\title{
Cytotoxicity of naphthoquinones and their capacity to generate reactive oxygen species is quenched when conjugated with gold nanoparticles
}

\author{
This article was published in the following Dove Press journal: \\ International Journal of Nanomedicine \\ 22 September 2011 \\ Number of times this article has been viewed
}

\author{
Priya Srinivas ${ }^{1,2}$ \\ Chitta Ranjan Patra ${ }^{2,4}$ \\ Santanu Bhattacharya ${ }^{2}$ \\ Debabrata Mukhopadhyay ${ }^{2,3}$ \\ 'Integrated Cancer Research \\ Program, Rajiv Gandhi Centre \\ for Biotechnology, Kerala, India; \\ ${ }^{2}$ Department of Biochemistry and \\ Molecular Biology; ${ }^{3}$ Department of \\ Biomedical Engineering, Mayo Clinic \\ College of Medicine, Rochester, \\ MN, USA; ${ }^{4}$ Department of Chemical \\ Biology, Indian Institute of Chemical \\ Technology, Hyderabad, India
}

Correspondence: Priya Srinivas Integrated Cancer Research Program,

Rajiv Gandhi Centre for Biotechnology,

Thycaud, PO 695014, Thiruvananthapuram,

Kerala, India

Tel 9| 47 I 2347975

Fax 9I 47 I2348096

Email priyasrinivas@rgcb.res.in

\begin{abstract}
Several reports have demonstrated the anticancer activities of plumbagin, a naphthoquinone derivative isolated from plants belonging to Plumbaginaceae family. However, to the best of our knowledge, there are no reports which describe gold nanoconjugation with plumbagin, even though plumbagin is considered to be a promising therapeutic agent. In this report, we demonstrate the fabrication and characterization of gold nanoparticles conjugated with plumbagin (AuPB) that can reduce the toxicity of the latter, and their capacity for cellular localization and generation of reactive oxygen species. The anticancer activity and ability of plumbagin to produce reactive oxygen species was studied and compared with that of bromoderivatives of 1,4 naphthoquinones such as 2-bromo-1,4-naphthoquinone (2-BNQ) and 2,3-dibromo-1, 4-naphthoquinone (2,3-DBNQ) and their gold nanoconjugates. Plumbagin and bromoderivatives of 1,4 naphthoquinones in the form of gold nanoconjugates showed reduced cytotoxicity and apoptosis compared with the pristine compounds, ie, plumbagin, 2-BNQ, and 2,3-DBNQ. Interestingly, we observed that the gold nanoparticles could quench the reactive oxygen speciesgenerating capacity of plumbagin, 2-BNQ, and 2,3-BNQ, which is one of the main mechanisms of action of the naphthoquinones. Therefore, it can be concluded that conjugation with gold nanoparticles can reduce the cytotoxicity of these compounds.
\end{abstract}

Keywords: plumbagin, gold nanoparticles, reactive oxygen species, naphthoquinones, breast cancer

\section{Introduction}

In Indian Ayurvedic medicine, plants belonging to Plumbaginaceae family, which are a rich source of plumbagin (5-hydroxy-2-methyl-1,4-naphthoquinone), have been used to treat a wide variety of ailments. Research in the last two decades has suggested that plumbagin is associated with a range of potential therapeutic or preventive effects. ${ }^{1-4}$ Though several studies have reported the anticancer activity of plumbagin, it is relatively nontoxic to normal cells..$^{5-9}$ The mechanism of action of plumbagin involves generation of reactive oxygen species, such as superoxide radicals $\left(\mathrm{O}_{2}^{-}\right)$and hydrogen peroxide $\left(\mathrm{H}_{2} \mathrm{O}_{2}\right)$, in the system. ${ }^{10,11}$ Thus, plumbagin has also been widely used as a model for studies of oxidative damage. ${ }^{12}$ The hydroxyl group of plumbagin increases its electrophilic properties and makes it a more potent cytotoxic molecule via its ability to accept electrons, which increases its capacity to generate reactive oxygen species. Although plumbagin has been shown to be more toxic to cancer cells, it can also be toxic to normal cells at high concentrations. ${ }^{13}$ In this context, nanotechnologies offer 
an exciting range of new therapeutic possibilities for cancer treatment.

In cancer nanotechnology, inorganic nanoparticles, especially gold nanoparticle-mediated drug delivery, might significantly reduce the drug doses needed, with high specificity, low toxicity, and better bioavailability. Recently, several groups ${ }^{14-16}$ including ours, have demonstrated that inorganic nanoparticles, especially gold nanoparticles (AuNP), possess enormous potential to improve the efficacy of several cancer treatments. ${ }^{17-19}$ Colloidal AuNP have been used widely for biomedical diagnostics, drug delivery, and therapeutic applications in cancer. AuNP were chosen for our study because they have several advantages, including ease of synthesis, easy characterization due to the presence of a surface plasmon resonance band, a very high surface area, stability, biocompatibility, and surface functionalization. Gold is chemically inert but is highly reactive and catalytic at the nanoscale level due to its high surface to volume ratio and quantum size effect. Spherical AuNP (size $5 \mathrm{~nm}$ ) shows a single strong surface plasmon resonance absorption band in the visible region around $512 \mathrm{~nm}$, which has significant biological importance. ${ }^{18-20}$

In the present study, we demonstrated conjugation and characterization of gold nanoconjugates with plumbagin (Au-PB) and bromoderivatives of naphthoquinone, their cytotoxicity, localization, and capacity to generate reactive oxygen species. We also showed that the pristine compounds could induce apoptosis more effectively than the gold conjugates. We also observed that plumbagin, but not gold nanoconjugates with plumbagin and polyethylene glycol (PEG)-amine (Au-PAM-PB), could inhibit p53 protein expression because plumbagin specifically inhibits acetylation of p53, which makes it susceptible to ubiquitination. We also analyzed bromoderivatives of 1,4 naphthoquinones (2-bromo1,4-naphthoquinone [2-BNQ] and 2,3-bibromo-1,4naphthoquinone [2,3-DBNQ]) to see if they could have a better conjugation effect with AuNP or show any change in cytotoxicity on AuNP conjugation when compared with $\mathrm{Au}-\mathrm{PB}$. To the best of our knowledge, there are no reports that describe gold nanoconjugation with plumbagin for use as a therapeutic agent in cancer.

\section{Materials and methods Synthesis of AuNP}

AuNP were synthesized by wet chemical methods using tetrachloroauric acid ( $\mathrm{HAuCl}_{4}$, Aldrich Chemicals, St Louis, $\mathrm{MO})$ and sodium borohydride $\left(\mathrm{NaBH}_{4}\right.$, Aldrich Chemicals) as reducing agents, as described elsewhere. ${ }^{17,18}$ Briefly, an aqueous solution of $\mathrm{HAuCl}_{4}$ was mixed with an aqueous solution of $\mathrm{NaBH}_{4}$ and mixed under vigorous stirring for about 16 hours. AuNP were then characterized by several physicochemical techniques, including ultraviolet-visible spectroscopy and transmission electron microscopy.

\section{Conjugation of plumbagin, 2-BNQ, and 2,3-DBNQ with AuNP}

Initially we tried to conjugate plumbagin, 2-BNQ, and 2,3-DBNQ (Sigma-Aldrich, St Louis, MO) with colloidal AuNP directly by a single incubation step. Different concentrations of these compounds were added to the AuNP solution and stirred vigorously for one hour. The solutions were ultracentrifuged at $18,000 \mathrm{rpm}$ for one hour at $10^{\circ} \mathrm{C}$ in an OTD80B Sorvall ultracentrifuge (Thermofisher Scientific Inc, Waltham, MA) using a 502 Ti rotor. The resulting loose pellets were used for further characterization and finally for in vitro cell culture experiments.

\section{Conjugation of plumbagin with AuNP via PEG}

Several PEG backbones of sulfhydryl and PAM chemicals, like PEG disulfhydryl 2000, PEG tetrasulfhydryl 20,000, PEG disulfhydryl 10,000, methyl PEG sulfhydryl 2000, PEG-amine 10,000, and PEG-diamine 2000 (SunBio Corporation, Orinda, CA) were used to make Au-PEG-PB nanoconjugates. In the first step, the PEG molecules were incubated with AuNP at room temperature for one hour to prepare AuNP-PEG, and subsequently incubated with plumbagin for another hour under similar conditions to make AuNP-PEG-PB in line with our previously published work. ${ }^{20}$ Finally, the nanoconjugates were centrifuged at 18,000 rpm for one hour at $10^{\circ} \mathrm{C}$, and the loose pellets and supernatant were analyzed for further characterization and in vitro cell culture experiments.

\section{Ultraviolet spectroscopy}

Absorption of the AuNP was measured using a Shimadzu spectrophotometer (Shimadzu Scientific Instruments Inc, Columbia, MD) to identify their ultraviolet-visible spectra (from 800 to $200 \mathrm{~nm}$ with a resolution of $1 \mathrm{~nm}$ ).

\section{Release of plumbagin from nanoconjugates}

The release study for the gold nanoconjugates was done as described elsewhere. ${ }^{20}$ Briefly, the original pellets of gold nanoconjugates (Au-PEG-PB) were divided into several equal fractions, diluted with equal volumes of phosphatebuffered solution ( $\mathrm{pH}$ 7.4), and incubated for different 
time periods $(0,0.30,1,2,4$, and 6 hours $)$ at $37^{\circ} \mathrm{C}$. All the fractions were centrifuged at $15,000 \mathrm{rpm}$ for one hour at $4^{\circ} \mathrm{C}$ after incubation. The supernatant was collected from each fraction and we then measured the absorbance of plumbagin using ultraviolet spectroscopy.

\section{In vitro cytotoxicity studies with MDA-MB-23 I cells}

In this study, we used MDA-MB-231 cells (American Type Culture Collection) as a model of breast cancer cells. Cells were maintained in Dulbecco's modified Eagle's medium with $10 \%$ fetal calf serum and $1 \%$ antibiotics in a humidified $5 \% \mathrm{CO}_{2}$ incubator at $37^{\circ} \mathrm{C}$. In order to check the functional activity of plumbagin and its derivatives, the cells were incubated for different periods of time and with different concentrations of the study compounds, as appropriate for such experiments.

\section{MTS and thymidine incorporation assays}

An in vitro cell proliferation assay was done using a colorimetric 3-(4-5- dimethylthiazol-2-yl)-5-(3-carboxymethoxyphenyl)-2(4-sulfophenyl)-2H-tetrazolium salt (MTS) assay (Promega, Madison, WI). Briefly, the cells were treated with $100 \mathrm{nM}$ of the gold nanoconjugates or the pristine compounds (plumbagin, 2-BNQ, and 2,3-DBNQ) in a 96-well plate (10,000 cells/well) for 48 hours, and the experiment was performed as described previously. ${ }^{21}$ Thymidine incorporation was also done to measure the growth proficiency of the cells. Briefly, after 48 hours of treatment with the compounds, $2.4 \times 10^{5}$ cells in a 24 -well plate were treated with ${ }^{3} \mathrm{H}$ thymidine for four hours $(0.5 \mu \mathrm{Ci}$, in RPMI with $10 \%$ fetal bovine serum per well). After washing with ice-cold phosphate-buffered solution, the proteins were fixed by adding $1 \mathrm{~mL}$ of ice-cold methanol into each well and incubated for 15 minutes at room temperature. The cells were then washed with water, and $300 \mu \mathrm{L}$ of $0.2 \mathrm{~N} \mathrm{NaOH}$ was then added to each well and incubated for 30 minutes. Two milliliters of scintillation fluid was added, and the radioactivity was counted using a liquid scintillation counter. The extent of thymidine incorporation was measured as the growth proficiency of the cells.

\section{Apoptosis by Annexin $\mathrm{V}$ binding assay}

After treating the cells for 24 hours with $100 \mathrm{nM}$ of the pristine compounds (plumbagin, 2-BNQ, or 2,3-DBNQ), Au-2-BNQ, Au-2,3-DBNQ), Au-PEG-PB, or AuNP alone were dispersed by trypsin-ethylenediamine tetra-acetic acid and washed further with $1 \times$ phosphate-buffered solution. Early apoptotic events were then determined using the ApoAlert
Annexin V kit (Clontech, Palo Alto, CA) as described by the manufacturer. Briefly, five million cells were resuspended in $200 \mu \mathrm{L}$ of $1 \times$ binding buffer containing Annexin V $0.1 \mu \mathrm{g} / \mathrm{mL}$ and propidium iodide $0.5 \mu \mathrm{g} / \mathrm{mL}$. The cells were analyzed by flow cytometry. Flow cytometric quantification of DNA was performed using a FACScan (Becton Dickinson, San Jose, CA) and data analysis was carried out using Modfit software.

\section{Western blot analysis}

The cells were incubated with the $100 \mathrm{nM}$ conjugates for 24 hours. The whole cell lysate, including the floating cells (total eight million cells), was collected in RIPA buffer supplemented with a protease inhibitor cocktail. Protein $10 \mu \mathrm{g}$ was loaded for blotting. Mouse monoclonal antibodies, p53 (DO-1, SC-126, Santa Cruz Biotechnology, Santa Cruz, CA) and $\beta$-actin (Sigma) were incubated overnight at $4{ }^{\circ} \mathrm{C}$ and used for immunodetection. After washing the membrane with $1 \times$ phosphate-buffered solution, the membrane was incubated with horseradish peroxidase-conjugated secondary antibody (antimouse IgG, Santa Cruz Biotechnology) for 45 minutes. The bands were visualized as per the protocols given in the West Pico chemiluminescence detection kit and the Super Signal West Pico substrate (Pierce Biotechnology, Rockford, IL).

\section{Transmission electron microscopy}

About one million cells were incubated with $1 \mu \mathrm{MAu}-\mathrm{PAM}-\mathrm{PB}$ for 24 hours. After treatment, the cells were trypsinized and resuspended in Trump's fixative containing 1\% glutaraldehyde and 4\% formaldehyde in $0.1 \mathrm{M}$ phosphate buffer at $\mathrm{pH}$ 7.2 and processed as already described in the literature. ${ }^{19,22}$ Thin (90 nm) sections were cut using a Reichert Ultra cut E ultramicrotome, placed on 200 mesh copper grids, and stained with lead citrate. Micrographs were taken on a TECNAI 12 operating at $120 \mathrm{kV}$.

\section{Spectrofluorometric analysis of reactive oxygen species}

The cells were seeded at a concentration of 10,000 cells per well in a 96-well plate. After overnight incubation, the cells were treated with $100 \mathrm{nM}$ of different AuNP/compounds $/ \mathrm{H}_{2} \mathrm{O}_{2}$ $10 \mu \mathrm{M}$ in serum-free Dulbecco's modified Eagle's medium for 30 minutes. Cells were then washed and resuspended in phosphate-buffered solution and incubated with $5 \mu \mathrm{M} 2^{\prime}, 7^{\prime}$ dichlorofluorescin diacetate $\mathrm{H}_{2}$ (Molecular Probes, Invitrogen, Carlsbad, CA) in ethanol for 30 minutes in the dark. Readings were taken on a Shimadzu UV PC2101 (Shimadzu Scientific Instruments Inc) at $495 \mathrm{~nm}$ excitation and $523 \mathrm{~nm}$ emission. 


\section{Results and discussion}

\section{Plumbagin conjugated with PEG-diamine 2000-coated AuNP}

Characterizations of AuNP formed by the $\mathrm{NaBH}_{4}$ reduction method were performed using ultraviolet-visible spectroscopy which showed a characteristic surface plasmon resonance band at $512 \mathrm{~nm}$, suggesting the formation of spherical AuNP. Plumbagin, 2-BNQ, and 2,3-DBNQ showed absorbance peaks at 420,350, and $360 \mathrm{~nm}$, respectively. To analyze whether the plumbagin, 2-BNQ, and 2,3-DBNQ had conjugated with AuNP, the loose pellets collected after centrifugation were analyzed by ultraviolet spectroscopy. We found that 2-BNQ and 2,3-DBNQ conjugated directly with AuNP. This may have been due to the bromine side chain in naphthoquinone where the bromo group can make a slight positive charge in the benzene ring, enabling weak bond formation between the positively charged benzene ring and the slight negatively charged AuNP. Therefore, 2-BNQ and 2,3-DBNQ can conjugate with AuNP. Another report suggests that this may be due to the bromine side chain which can bond with AuNP directly, because bromide ions of bromoderivatives can replace the chloride ions in $\mathrm{AuCl}_{4}{ }^{23} \mathrm{~A}$ 2-BNQ concentration of $20 \mu \mathrm{g} / \mathrm{mL}$ was used for further studies because maximum absorbance at $350 \mathrm{~nm}$ was observed at this concentration (Figure 1A). The same concentration was chosen for 2,3-DBNQ because the absorption at $520 \mathrm{~nm}$ was maximum at $20 \mu \mathrm{g} / \mathrm{mL}$ (Figure 1B). On the other hand, AuNP with plumbagin did not show any absorbance at $420 \mathrm{~nm}$, indicating that direct conjugation of plumbagin with AuNP had not taken place. The maximum number of naphthoquinones was attached to the AuNP at $20 \mu \mathrm{g} / \mathrm{mL}$, beyond which aggregation took place instead of binding more molecules to the AuNP. The decrease in absorbance indicates aggregation of AuNP in line with our previously published work. ${ }^{19,20}$

In this context, we tried to conjugate plumbagin with AuNP through various sulfhydryl and amine derivatives of PEG molecules, whereby PEG was used as a linker because plumbagin did not conjugate directly with AuNP. Among several PEG molecules (discussed in the Material and methods section), PEG-diamine 2000 was found to be conjugated with AuNP, so we used PAM for surface functionalization of AuNP, after which plumbagin was conjugated with AuNP without any aggregation and showed absorbance at $420 \mathrm{~nm}$ (Figure $1 \mathrm{C}$ ), where $15 \mu \mathrm{g} / \mathrm{mL}$ of PAM was used for conjugation with AuNP. ${ }^{20}$ The dependence on absorption of the conjugates at $420 \mathrm{~nm}$ indicated that a concentration ratio of $30 \mu \mathrm{g} / \mathrm{mL}$ (with respect to $1 \mathrm{~mL}$ of AuNP-PAM solution) of plumbagin could be used for further experiments. The change of absorption maxima $\left(\lambda_{\max }\right)$ of AuNP upon addition of PEG-PB, 2-BNQ, or 2,3-DBNQ, as compared with the spectrum for AuNP alone $\left(\lambda_{\text {max }}=512 \mathrm{~nm}\right)$ clearly demonstrates a red shift in the surface plasmon resonance band maxima in Au-PEG-PB $\left(\lambda_{\text {max }}=536 \mathrm{~nm}\right)$, Au-2-BNQ ( $\left.\lambda_{\text {max }}=542\right)$, and Au-2,3-DBNQ $\left(\lambda_{\text {max }}=535\right)$, respectively (Figure $2 \mathrm{~A}-\mathrm{C}$ ). Addition of PAM compounds to AuNP caused red shifts in the $\lambda_{\max }$ values, suggesting interaction and binding of the individual components with the surface of the negatively charged AuNP. Therefore, further experiments were done with the Au-2-BNQ, Au-2,3-DBNQ, and Au-PAM-PB complexes. According to the published literature, we hypothesized that the naphthoquinone-derived AuNP conjugate concept is based on a combination of the favorable properties of the AuNP and the naphthoquinone molecules when forming a naphthoquinone-gold nanoparticle assembly. ${ }^{24-26} \mathrm{~A}$ predicted schematic diagram of the Au-PAM-PB complex is shown in Figure 2D. According to our earlier work, the absorption peak at $512 \mathrm{~nm}$ indicates the spherical nature of the nanoparticles. The high wavelength indicates an association with AuNP, which increases the stability of the nanoconjugate and the hydrodynamic radius of the particles. ${ }^{18}$ The release kinetics of plumbagin from the Au-PAM-PB nanoconjugates was also analyzed, and a constant release of plumbagin was observed for up to 24 hours (Figure 3).

\section{AuNP-conjugated compounds may reduce cytotoxicity and apoptosis}

MTS assay showed that AuNP-conjugated plumbagin, 2-BNQ, and 2,3-DBNQ (Au-2-BNQ, Au-2,3-DBNQ, and Au-PAM-PB) could reduce antiproliferative effects to the same extent as the pristine compounds (Figure 4A and B). Analysis of the number of cells in the $\mathrm{S}$ phase using the thymidine incorporation assay showed similar results. Changes in the phospholipid bilayer of the cell membrane were also observed early in apoptosis. The phosphatidylserine component of the phospholipid bilayer was externalized and could be detected by fluorescence labeling. Annexin $\mathrm{V}$ has a high affinity for the phosphatidylserine-containing phospholipid bilayer. Staining with FITC-conjugated Annexin $\mathrm{V}$ and propidium iodide can identify subpopulations of cells with membrane changes and any associated loss of membrane integrity. In this study, further apoptotic induction studies by Annexin-propidium iodide staining confirmed that the AuNP-conjugated molecules could reduce apoptosis 

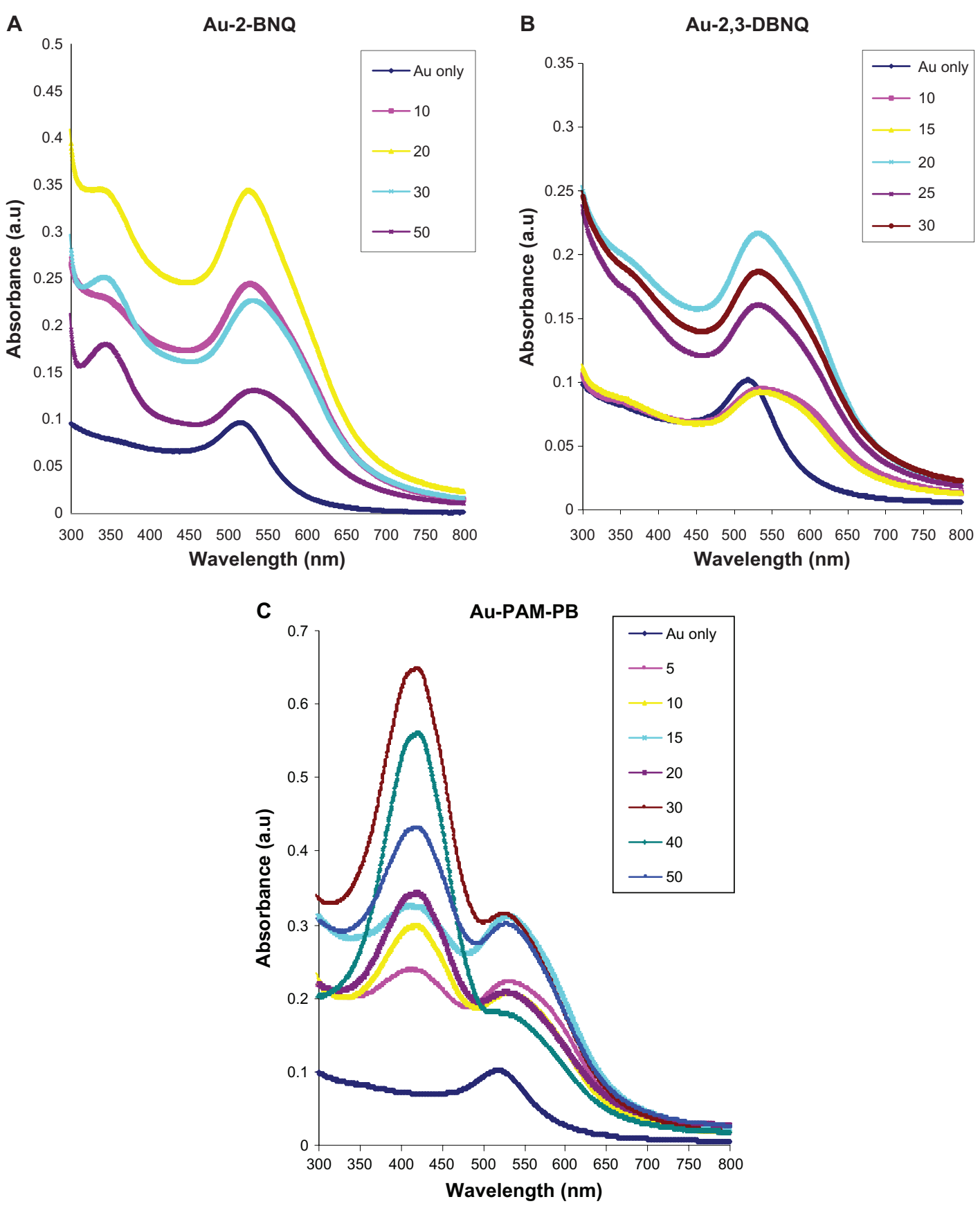

Figure I Ultraviolet-visible spectra of Au-2-BNQ, Au-2,3-DBNQ, and Au-PAM-PB nanoconjugates. Using different concentrations of conjugates (I0 $\mu \mathrm{g} / \mathrm{mL}$ of AuNP solution), an increase in absorbance of (A) Au-2-BNQ at $350 \mathrm{~nm}$ and (B) Au-2,3-DBNQ at $360 \mathrm{~nm}$ is observed with increasing concentrations of 2-BNQ (10,20,30, and 50 $\mu \mathrm{g} / \mathrm{mL})$ and 2,3-DBNQ (10, 15, 20, 25 and $30 \mu \mathrm{g} / \mathrm{mL})$, respectively, up to $20 \mu \mathrm{g} / \mathrm{mL}$ is seen, and then a decrease. Absorbance increases up to $20 \mu \mathrm{g} / \mathrm{mL}$ and then the absorbance decreases with increasing concentrations of both 2-BNQ and 2,3-DBNQ. (C) Similarly, imilarly, for Au-PAM-PB (5, 10, 15, 20, 30, 40, and $50 \mu \mathrm{g} / \mathrm{mL}$ ), the absorbance at $420 \mathrm{~nm}$ increases to a maximum at $30 \mu \mathrm{g} / \mathrm{mL}$ and then decreases with an increase in concentration of plumbagin. All experiments were performed three times with similar results.

Abbreviations: Au, gold; 2-BNQ, 2-bromo-I,4-naphthoquinone; 2,3-DBNQ, 2,3-dibromo-I,4-naphthoquinone; PB, plumbagin; PAM, polyethylene glycol-amine.

more than the pristine compounds alone (Figure 5). Similar results showing the inhibitory effect of anisamide-targeted liposomal doxorubicin on the growth of DU-145 tumors were reported by Banerjee et al, who showed that despite the improved targeting, doxorubicin formulated in liposomes containing distearoylglycerol phosphatidylethanolamine polyethylene glycol(3400)- -[2-(4-methoxybenzamido)] ethylamine were less potent than the free drug. This might be due to the fact that the tumor cells were exposed to the free drug for an extended period of time. ${ }^{27}$ In vivo, this condition might not occur as the free drug is rapidly cleared after administration. 
A

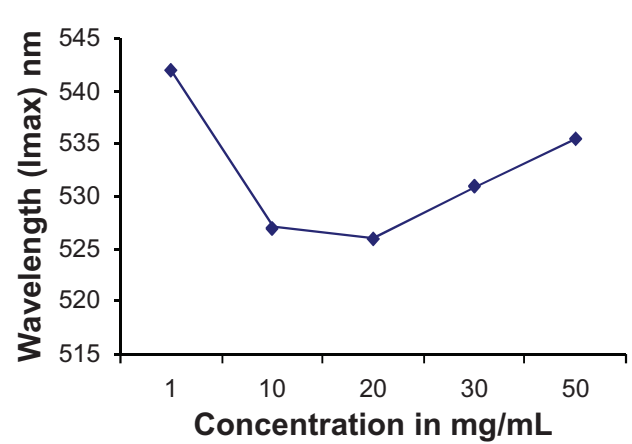

C

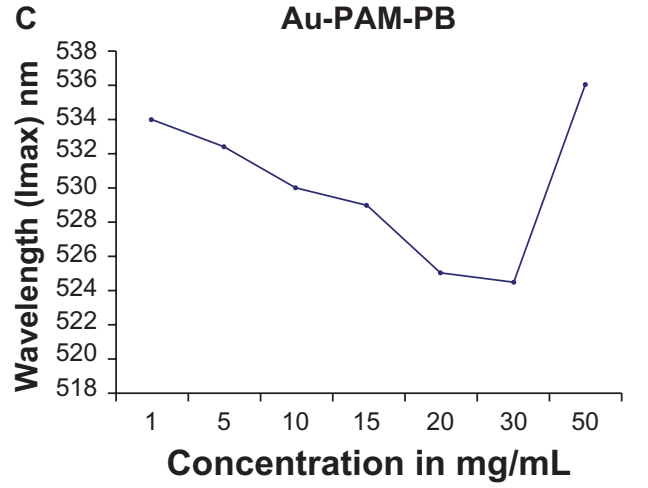

B

Au-2,3-DBNQ- $\lambda$ max

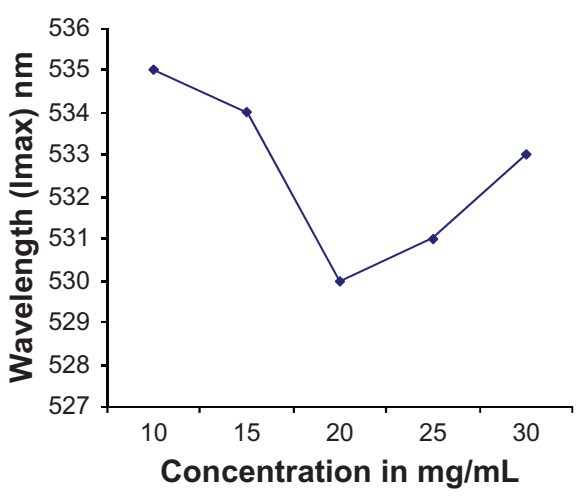

D

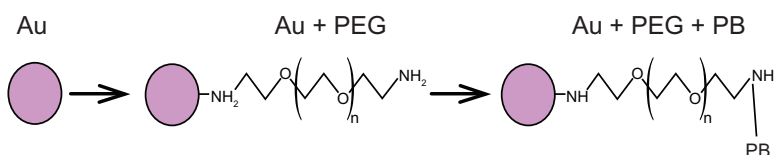

Figure 2 Ultraviolet-visible spectral readings of AuNP and nanoconjugates indicating the change in peak of wavelength (shift in $\lambda$ ) of gold nanoparticles with increasing concentrations of (A) 2-BNQ, (B) 2,3-DBNQ, and (C) Au-PAM-PB. A predicted schematic diagram of Au-PAM-PB complex is shown in (D). Au-PAM may conjugate with plumbagin by amide bonding.

Abbreviations: Au, gold; 2-BNQ, 2-bromo-I,4-naphthoquinone; 2,3-DBNQ, 2,3-dibromo-I,4-naphthoquinone; PB, plumbagin; PAM, polyethylene glycol-diamine.

\section{p53 and apoptosis}

Apoptosis can be p53-dependent or p53-independent. Plumbagin is reported specifically to inhibit p300-mediated acetylation of $\mathrm{p} 53$, making it susceptible to ubiquitination. The chemical entity in plumbagin responsible for inhibition of acetyltransferase activity is the hydroxyl group. ${ }^{28}$
Here we observed that treatment with plumbagin but not with Au-PAM-PB could inhibit p53 protein expression (Figure 6). 2-BNQ and 2,3-DBNQ and their gold conjugates did not inhibit p53. This might be due to the lack of a hydroxyl group in these quinones. This indirectly shows that AuNP can change the electrophilicity of the hydroxyl group on plumbagin.

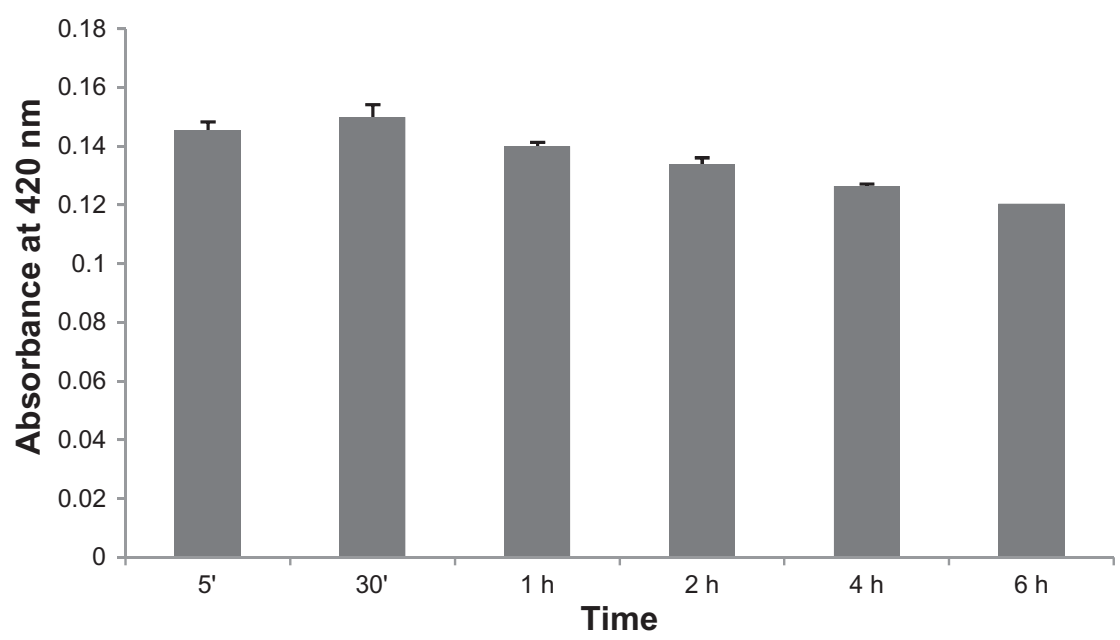

Figure 3 Release profiles for plumbagin from Au-PAM-PB nanoconjugates. There was a constant release of plumbagin from Au-PAM-PB for up to six hours in phosphatebuffered solution at $\mathrm{pH} 7.4$.

Abbreviations: Au, gold; PB, plumbagin; PAM, polyethylene glycol-amine. 

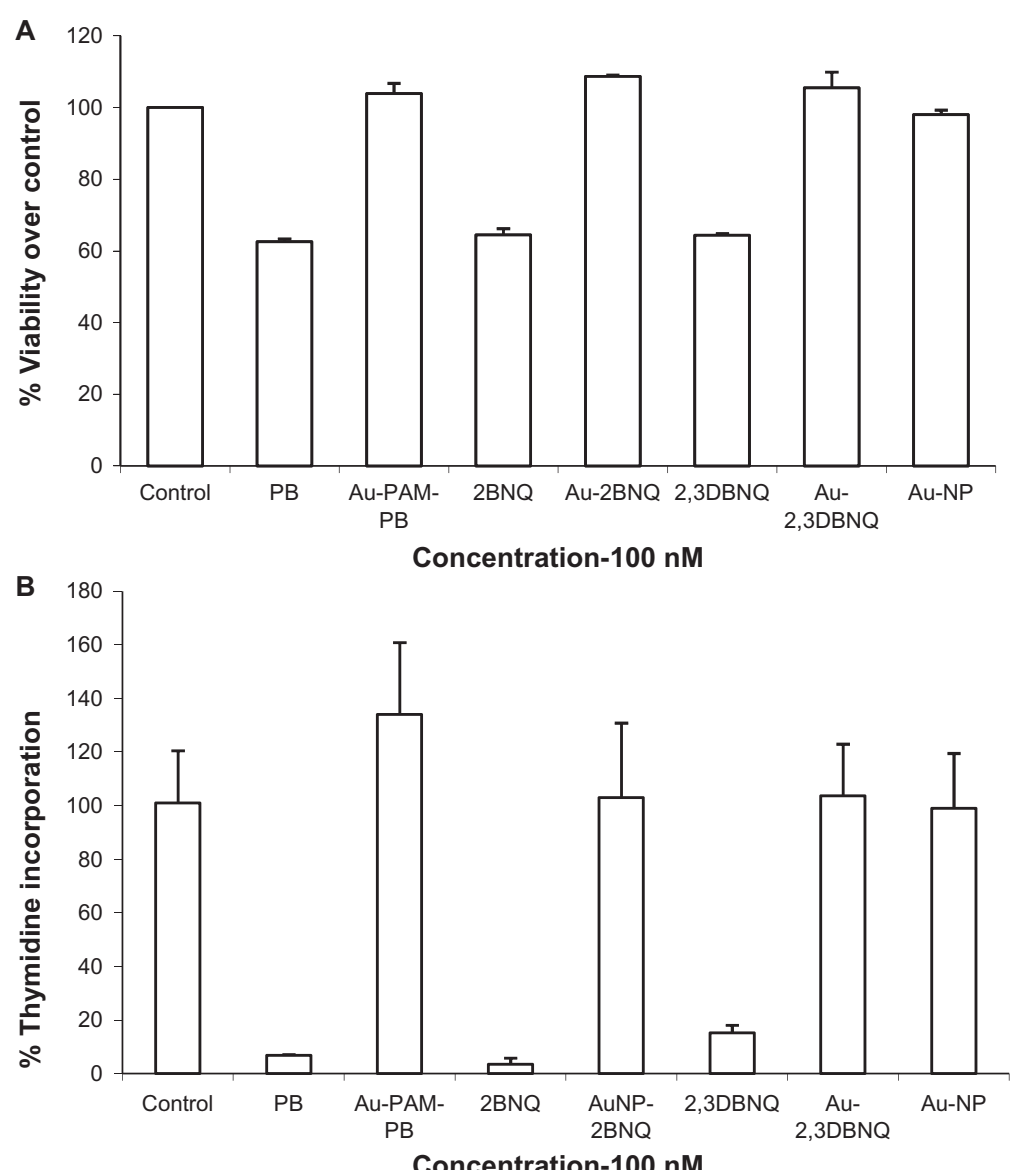

Figure 4 (A) MTS cell proliferation assay with naphthoquinones in MDA-MB-23I cells. Concentrations of all free compounds and compounds with AuNP conjugates were constant ( $100 \mathrm{nM})$ as indicated. The concentration was calculated from the standard curve drawn using the absorbance obtained at 420, 350, and $360 \mathrm{~nm}$ for plumbagin, 2-BNQ, and 2,3-DBNQ, respectively. MTS reading was taken after 48 hours of incubation with the compounds and AuNP conjugates. (B) Thymidine incorporation assay also showed fewer $\mathrm{S}$ phase cells in those treated with the pristine compound compared than the AuNP conjugates. The experiment was repeated at least three times, with similar results. Abbreviations: Au, gold; 2-BNQ, 2-bromo-I,4-naphthoquinone; 2,3-DBNQ, 2,3-dibromo-I,4-naphthoquinone; PB, plumbagin; NP, nanoparticles.

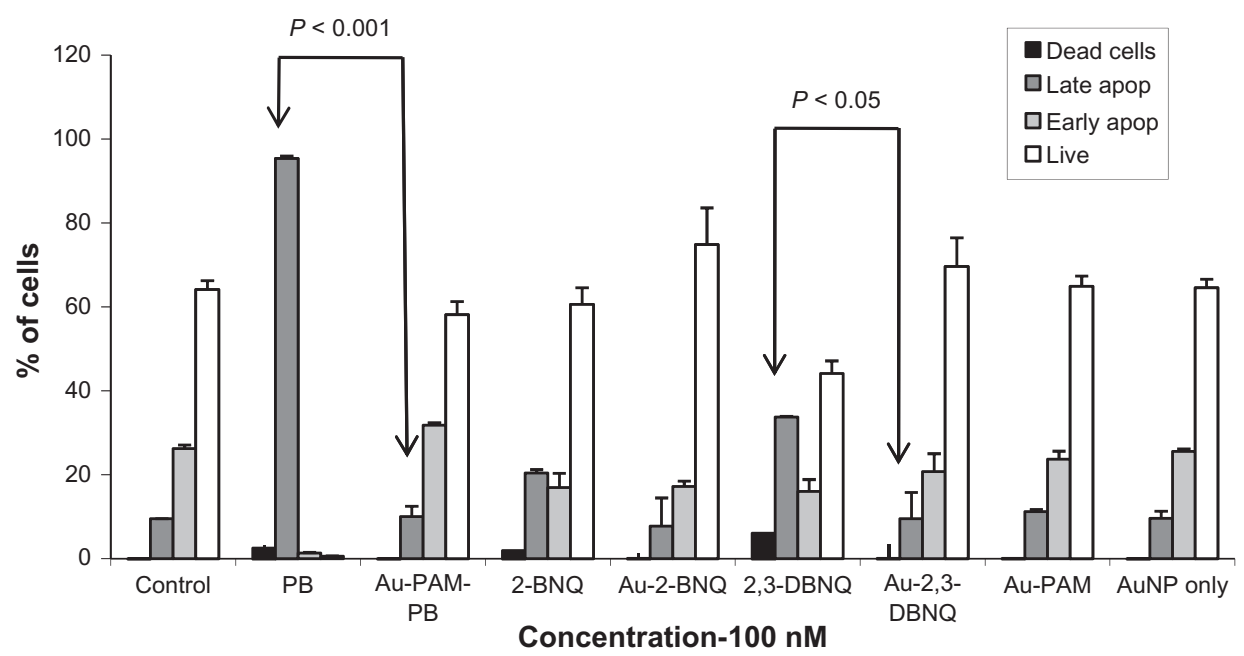

Figure 5 Cells were counted after Annexin-propidium iodide staining. Cells with bound Annexin $\vee$ show green staining in the plasma membrane. Cells that have lost membrane integrity show red staining (propidium iodide) throughout the cytoplasm. Flow cytometric analysis showed live (no stain), early apoptotic cells (only annexin staining), late apoptotic cells (both annexin and propidium iodide staining), and dead (propidium iodide staining) cells. Most of the plumbagin-treated cells were in the late apoptotic stage, whereas the AuNP conjugates had fewer numbers of late apoptotic cells. More live cells are observed in AuNP conjugates than in samples treated with the pristine compound. A $t$-test showed a statistically significant difference between the percentage of late apoptotic cells induced by plumbagin versus Au-PAM-PB and $2,3-$ DBNQ versus Au-2,3-DBNQ. The experiment was repeated at least three times with similar results.

Abbreviations: Au, gold; 2-BNQ, 2-bromo-I,4-naphthoquinone; 2,3-DBNQ, 2,3-dibromo-I,4-naphthoquinone; PB, plumbagin; PAM, polyethylene glycol-amine; NP, nanoparticles. 


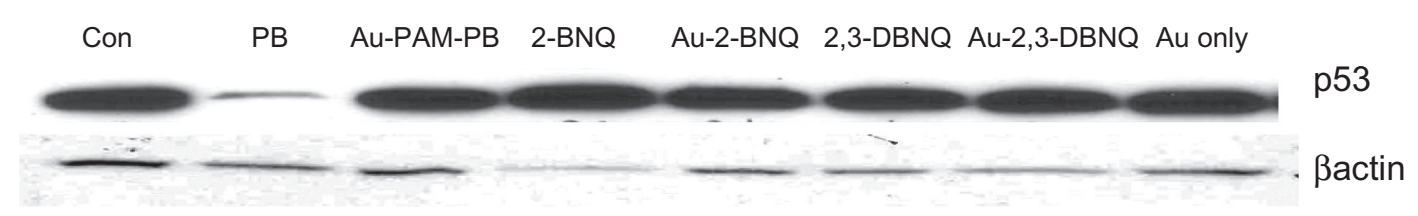

Figure 6 Western blot analysis of p53 protein expression showed that only plumbagin-treated cells had reduced expression of $\mathrm{p} 53$ at $100 \mathrm{nM}$ concentration.

\section{Au-PAM-PB aggregates in lysosomes}

MDA-MB-231 cells were treated with $1 \mu \mathrm{M}$ Au-PAM-PB for 24 hours, and their localization inside the cells was checked by transmission electron microscopy, revealing uptake of synthesized AuNP in vacuoles and lysosomes in the cytoplasmic compartment, as shown in Figure 7.

A

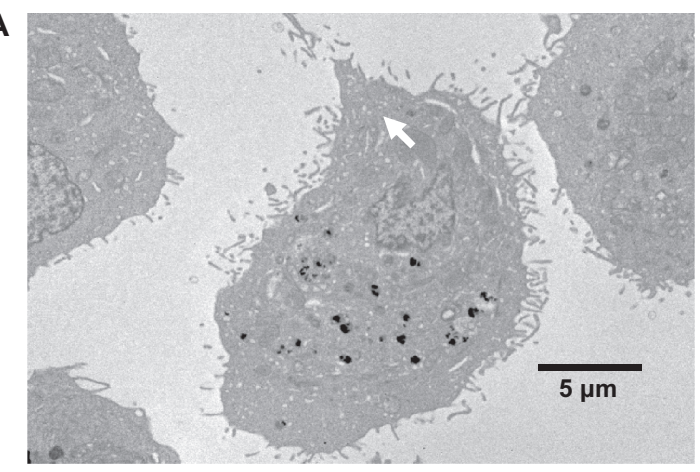

B

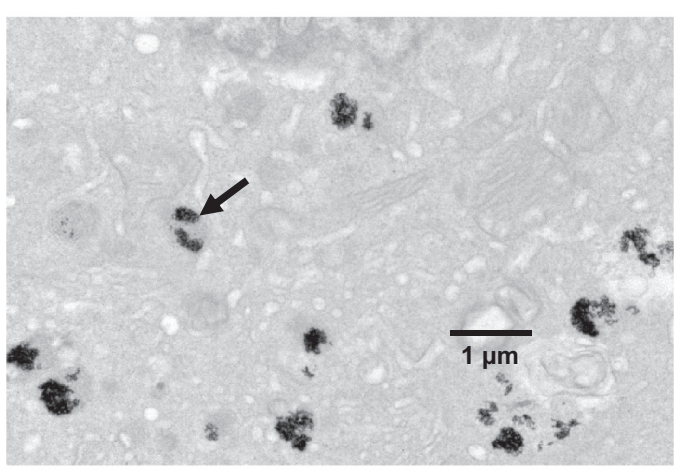

C

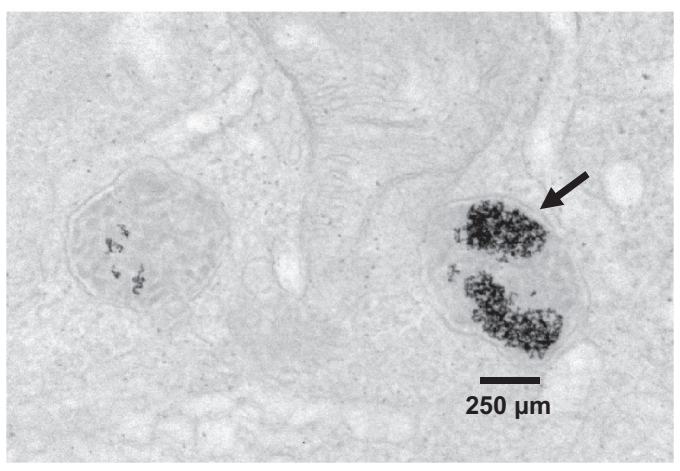

Figure 7 Cellular uptake of Au-PAM-PB. Transmission electron microscopic analysis of MDA-MB-23I cells treated with Au-PAM-PB $100 \mathrm{nM}$ for 24 hours showed internalization of Au-PAM-PB by lysosomes in the cells. Magnifications of the figures shown as (A) 6000x, (B) 20,000x, and (C) 80,000x. The experiment was repeated at least three times with similar results.

Abbreviations: Au, gold; PB, plumbagin; PAM, polyethylene glycol-amine.
AuNP are reported to be internalized in the cell and to accumulate inside lysosomes. ${ }^{29}$ However, in this study, it was not analyzed whether the AuNP found in the lysosomes contained bound plumbagin or not.

\section{AuNP quenches production of reactive oxygen species by naphthoquinones}

The ability of AuNP-conjugated plumbagin to generate reactive oxygen species was analyzed because plumbagin is a well known generator of reactive oxygen species. ${ }^{10,11}$ There is extensive electron delocalization between the $\mathrm{C} 1$ to $\mathrm{C} 4$ carbon atoms, which is responsible for the reversible redox cycling observed for plumbagin (by electrochemical measurement) whereby it generates successive semiquinone and catecholic forms. The intermediate semiquinone formed is highly reactive and interacts with molecular dioxygen species present in biological systems to oxidize itself back to the original quinone form, while the molecular oxygen gets reduced to superoxide species. This is what is commonly known as "redox cycling" of quinones, and is responsible for most of their biological activity. As we observed quenching of the redox cycling behavior of plumbagin upon making an AuNP conjugate, such conjugation does not allow or favor addition of one electron in the lowest unoccupied molecular orbits. This could be due to substitution of electrons, withdrawing a chloro/bromo substituent at the $\mathrm{C} 3 / \mathrm{C} 2$ position or perturbation by the molecular orbits of gold in the quinone reduction. It has been reported that AuNP can cause an approximately 1.5-fold increase in reactive oxygen species levels in human hepatoma cells..$^{30}$ Another report by Ito et $\mathrm{a}^{31}$ showed that AuNP increase the yield of reactive oxygen species and that the antioxidant enzymes, catalase and superoxide dismutase, are both effective in reducing the total amount of reactive oxygen species..$^{31}$ This analysis was done using electron paramagnetic resonance spectroscopy in cell-free media. Other reports also show that AuNP can cause elevation of reactive oxygen species levels without cytotoxicity. ${ }^{32,33}$ On the other hand, quinones can undergo intracellular electron reduction to the hydroquinone or to the semiquinone. ${ }^{34,35}$ The semiquinone or hydroquinone may regenerate quinone through redox cycling by reacting with molecular oxygen to produce superoxide 


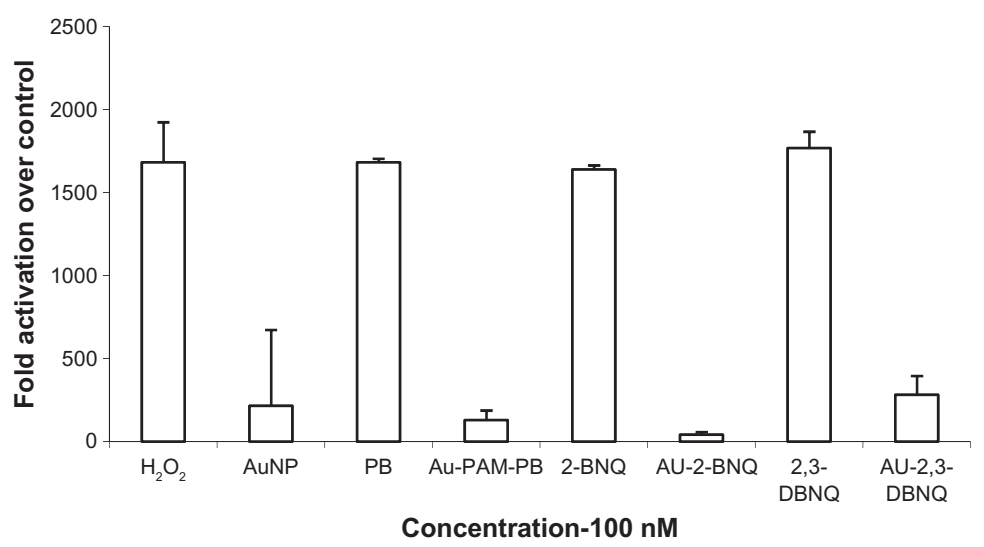

Figure 8 Reactive oxygen species generation study with DCFH-DAH $\mathrm{H}_{2}$ staining. $\mathrm{H}_{2} \mathrm{O}_{2}$ production was detected using nonfluorescent DCFH-DAH $\mathrm{H}_{2}$ dye, which when oxidized is converted into fluorescent $2^{\prime}, 7^{\prime}$-dichlorofluorescein (excitation $488 \mathrm{~nm}$, emission $540 \mathrm{~nm}$ ). The AuNP conjugates and AuNP alone could not induce reactive oxygen species production, while the positive control $\left(10 \mu \mathrm{M} \mathrm{H}_{2} \mathrm{O}_{2}\right)$ and the pristine compounds generated reactive oxygen species. The experiment was repeated at least three times with similar results.

Abbreviations: Au, gold; NP, nanoparticles.

radicals. This may culminate in damage to macromolecules and cytotoxicity. ${ }^{36}$ The production of oxygen radicals and the cytotoxic damage vary for different quinones and in different environments. ${ }^{37,38}$ Thus, both AuNP and naphthoquinones generate reactive oxygen species. However, AuNP-conjugated naphthoquinones might exhibit a relative antioxidant potential compared with the pristine compounds. It is known that antioxidant capacity is a relative phenomenon. A pro-oxidant may become antioxidant when it encounters a stronger prooxidant. Similarly, an antioxidant may become pro-oxidant depending on the redox potential. Therefore, interpretation of the antioxidant/pro-oxidant status of chemical entities should be done only after considering these issues. We had shown earlier that pretreatment of cells with $\mathrm{N}$-acetylcysteine, an antioxidant, protected cells from undergoing apoptosis, confirming the role of reactive oxygen species in plumbaginmediated apoptosis. ${ }^{39}$ Here we have found that the capacity of plumbagin to generate reactive oxygen species, which is the major mechanism of action, can be quenched by AuNP conjugation through PEG, which shows that cytotoxicity can be reduced by gold nanoconjugation (Figure 8). However, further studies need to be done to confirm this in vivo.

\section{Conclusion}

We have synthesized AuNP and their conjugates with plumbagin, 2-BNQ, and 2,3-DBNQ molecules and characterized them by several physicochemical techniques. We have also studied the anticancer activity of the free and gold nanoconjugated compounds. We have shown that the cytotoxicity of the free compounds can be reduced by conjugation with AuNP. A plausible mechanism might be less production of reactive oxygen species when these compounds are conjugated with
AuNP. The outcome of targeted chemotherapy depends mainly on two factors, ie, the ability of the carrier molecule to recognize malignant cells selectively and the nature of the chemical linkage used for coupling the cytotoxic agent to the carrier. Chemical bonding between plumbagin and AuNPPAM might affect the hydroxyl group of plumbagin, ie, the electrophilic moiety, making it a less toxic molecule, resulting in an ability to quench reactive oxygen species. Further studies of these AuNP-naphthoquinone conjugates with targeting antibodies or liposomes might enable these compounds to be administered with targeted cytotoxicity in a safe manner.

\section{Acknowledgment}

This study was supported by an overseas associateship grant to PS by the Department of Biotechnology, Government of India (San No. 102/IFD/SAN/415/2009-10). The authors are grateful to J Charlesworth from the Mayo Clinic for the transmission electron microscopic analysis. We acknowledge Dr Priyabrata Mukherjee, Department of Biochemistry and Molecular Biology, Mayo Clinic College of Medicine, and Professor Subhash Padhye from the Center for Drug Design and Molecular Medicine, Department of Environmental Sciences, University of Pune, India, for their kind suggestions for the study and preparation of the manuscript. CRP is grateful to the Department of Science and Technology, Govt of India, New Delhi for the award of Ramanujan Fellowship (SR/S2/RJN-04/2010, GAP 0305\DST \CP). This work was also supported by a generous gift to DM from Bruce and Martha Atwater.

\section{Disclosure}

The authors report no conflicts of interest in this work. 


\section{References}

1. Bhargava SK. Effects of plumbagin on reproductive function of male dog. Indian J Exp Biol. 1984;22:153-156.

2. de Paiva SR, Figueiredo MR, Aragão TV, Kaplan MA. Antimicrobial activity in vitro of plumbagin isolated from Plumbago species. Mem Inst Oswaldo Cruz. 2003;98:959-961.

3. Hsu YL, Cho CY, Kuo PL, Huang YT, Lin CC. Plumbagin (5-hydroxy-2methyl-1,4-naphthoquinone) induces apoptosis and cell cycle arrest in A549 cells through p53 accumulation via c-Jun NH2-terminal kinasemediated phosphorylation at serine 15 in vitro and in vivo. JPharmacol Exp Ther. 2006;318:484-494.

4. Itoigawa M, Takeya K, Furukawa H. Cardiotonic action of plumbagin on guinea-pig papillary muscle. Planta Med. 1991;57:317-319.

5. Chen CA, Chang HH, Kao CY, Tsai TH, Chen YJ. Plumbagin, isolated from Plumbago zeylanica, induces cell death through apoptosis in human pancreatic cancer cells. Pancreatology. 2009;9:797-809.

6. Demma J, Hallberg K, Hellman B. Genotoxicity of plumbagin and its effects on catechol and NQNO-induced DNA damage in mouse lymphoma cells. Toxicol In Vitro. 2009;23:266-271.

7. Jackson JK, Higo T, Hunter WL, Burt HM. Topoisomerase inhibitors as anti-arthritic agents. Inflamm Res. 2008;57:126-134.

8. Srinivas P, Gopinath G, Banerji A, Dinakar A, Srinivas G. Plumbagin induces reactive oxygen species, which mediate apoptosis in human cervical cancer cells. Mol Carcinog. 2004;40:201-211.

9. Thasni KA, Rakesh S, Rojini G, Ratheeshkumar T, Srinivas G, Priya S. Estrogen-dependent cell signaling and apoptosis in BRCA1-blocked BG1 ovarian cancer cells in response to plumbagin and other chemotherapeutic agents. Ann Oncol. 2008;19:696-705.

10. Xu KH, Lu DP. Plumbagin induces ROS-mediated apoptosis in human promyelocytic leukemia cells in vivo. Leuk Res. 2010;34:658-665.

11. Inbaraj JJ, Chignell CF. Cytotoxic action of juglone and plumbagin: a mechanistic study using HaCaT keratinocytes. Chem Res Toxicol. 2004; 17:55-62.

12. Burger RM, Drlica K. Superoxide protects Escherichia coli from bleomycin mediated lethality. J Inorg Biochem. 2009;103:1273-1277.

13. Ahmad A, Banerjee S, Wang Z, Kong D, Sarkar FH. Plumbagin-induced apoptosis of human breast cancer cells is mediated by inactivation of NF- $\mathrm{KB}$ and Bcl-2. J Cell Biochem. 2008;105:1461-1471.

14. Brown SD, Nativo P, Smith JA, et al. Gold nanoparticles for the improved anticancer drug delivery of the active component of oxaliplatin. J Am Chem Soc. 2010;132:4678-4684.

15. Paciotti GF, Myer L, Weinreich D, et al. Colloidal gold: a novel nanoparticle vector for tumor directed drug delivery. Drug Deliv. 2004;11: 169-183.

16. Li J, Wang X, Wang C, et al. The enhancement effect of gold nanoparticles in drug delivery and as biomarkers of drug-resistant cancer cells. Chem Med Chem. 2007;2:374-378.

17. Patra CR, Bhattacharya R, Wang E, et al. Targeted delivery of gemcitabine to pancreatic adenocarcinoma using cetuximab as a targeting agent. Cancer Res. 2008;68:1970-1978.

18. Patra CR, Bhattacharya R, Mukherjee P. Fabrication and functional characterization of goldnanoconjugates for potential application in ovarian cancer. J Mater Chem. 2010;20:547-554.

19. Das A, Mukherjee P, Singla SK, et al. Fabrication and characterization of an inorganic gold and silica nanoparticle mediated drug delivery system for nitric oxide. Nanotechnology. 2010;21:305102.
20. Bhattacharya R, Patra CR, Earl A, et al. Attaching folic acid on gold nanoparticles using noncovalent interaction via different polyethylene glycol backbones and targeting of cancer cells. Nanomedicine. 2007;3:224-238.

21. Wang L, Lau JS, Patra CR, et al. RGS-GAIP-interacting protein controls breast cancer progression. Mol Cancer Res. 2010;8:1591-1600.

22. Patra CR, Bhattacharya R, Patra S, et al. Proangiogenic properties of europium (III) hydroxide nanorods. Adv Mater. 2008;20:753-756.

23. Eustis S, El-Sayed MA. Molecular mechanism of the photochemical generation of gold nanoparticles in ethylene glycol: support for the disproportionation mechanism. J Phys Chem. 2006;110:14014-14019.

24. Miyachi M, Yamanoi Y, Shibata Y, et al. A photosensing system composed of photosystem I, molecular wire, gold nanoparticle, and double surfactants in water. Chem Commun. 2010;46:2557-2559.

25. Terasaki N, Yamamoto N, Tamada K, et al. Bio-photosensor: Cyanobacterial photosystem I coupled with transistor via molecular wire. Biochim Biophys Acta. 2007;176:653-659.

26. Quarta A, Di Corato R, Manna L, et al. Multifunctional nanostructures based on inorganic nanoparticles and oligothiophenes and their exploitation for cellular studies. J Am Chem Soc. 2008;130:10545-10555.

27. Banerjee R, Tyagi P, Li S, Huang L. Anisamide-targeted stealth liposomes: a potent carrier for targeting doxorubicin to human prostate cancer cells. Int J Cancer. 2004;112:693-700.

28. Ravindra KC, Selvi BR, Arif M, et al. Inhibition of lysine acetyltransferase KAT3B/p300 activity by a naturally occurring hydroxynaphthoquinone, plumbagin. J Biol Chem. 2009;284:24453-24464.

29. Hosta L, Pla-Roca M, Arbiol J, et al. Conjugation of Kahalalide F with gold nanoparticles to enhance in vitro antitumoral activity. Bioconjug Chem. 2009;20:138-146.

30. Fan JH, Hung WI, Li WT, Yeh J. Biocompatibility study of gold nanoparticles to human cells. IFMBE Proc. 2009;23:870-873.

31. Ito S, Miyoshi N, Degraff WG, Nagashima K, Kirschenbaum LJ, Riesz P. Enhancement of 5-aminolevulinic acid-induced oxidative stress on two cancer cell lines by gold nanoparticles. Free Radic Res. 2009;43:1214-1224.

32. Farkas J, Christian P, Urrea JA, Roos N, et al. Effects of silver and gold nanoparticles on rainbow trout (Oncorhynchus mykiss) hepatocytes. Aquat Toxicol. 2009;96:44-52.

33. Pan Y, Leifert A, Ruau D, et al. Gold nanoparticles of diameter $1.4 \mathrm{~nm}$ trigger necrosis by oxidative stress and mitochondrial damage. Small. 2009;5:2067-2076.

34. O'Brien PJ. Molecular mechanisms of quinone cytotoxicity. Chem Biol Interact. 1991;80:1-41.

35. Morgan WA, Hartley JA, Cohen GM. Quinone-induced DNA single strand breaks in rat hepatocytes and human chronic myelogenous leukaemic K562 cells. Biochem Pharmacol. 1992;44:215-221.

36. Ngo EO, Sun TP, Chang JY, et al. Menadione-induced DNA damage in a human tumor cell line. Biochem Pharmacol. 1991;42:1961-1968.

37. Rockwell S, Sartorelli AC, Tomasz M, Kennedy KA. Cellular pharmacology of quinone bioreductive alkylating agents. Cancer Metastasis Rev. 1993;12:165-176.

38. Gibson NW, Hartley JA, Butler J, Siegel D, Ross D. Relationship between DT-diaphorase-mediated metabolism of a series of aziridinylbenzoquinones and DNA damage and cytotoxicity. Mol Pharmacol. 1992;42:531-536.

39. Srinivas G, Annab LA, Gopinath G, Banerji A, Srinivas P. Antisense blocking of BRCA1 enhances sensitivity to plumbagin but not tamoxifen in BG-1 ovarian cancer cells. Mol Carcinog. 2004;39:15-25.
International Journal of Nanomedicine

\section{Publish your work in this journal}

The International Journal of Nanomedicine is an international, peerreviewed journal focusing on the application of nanotechnology in diagnostics, therapeutics, and drug delivery systems throughout the biomedical field. This journal is indexed on PubMed Central, MedLine, CAS, SciSearch ${ }^{\circledR}$, Current Contents $\AA /$ Clinical Medicine,

\section{Dovepress}

Journal Citation Reports/Science Edition, EMBase, Scopus and the Elsevier Bibliographic databases. The manuscript management system is completely online and includes a very quick and fair peer-review system, which is all easy to use. Visit http://www.dovepress.com/ testimonials.php to read real quotes from published authors. 